\title{
An Identity on Partial Generalized Automorphisms of Prime Rings
}

\author{
Shuliang HuAng (*)
}

ABSTRACT - Let $R$ be a prime ring with center $Z(R), T: R \longrightarrow R$ be a non-zero partial generalized automorphism of $R, L$ a Lie ideal of $R, s \geq 0, t \geq 0$ and $n \geq 1$ fixed integers, such that $\left(u^{s}(T(u) \circ u) u^{t}\right)^{n}=0$ for all $u \in L$. If either $\operatorname{Char}(R)>n+1$ or $\operatorname{Char}(R)=0$, then $L \subseteq Z(R)$.

Mathematics Subject Classification (2010). 16N60, 16U80, 16 W25.

KEYWORDs. Prime ring, Lie ideal, partial generalized automorphism.

\section{Introduction}

The standard identity $s_{4}$ in four variables is defined as follows:

$$
s_{4}=\sum(-1)^{\tau} X_{\tau(1)} X_{\tau(2)} X_{\tau(3)} X_{\tau(4)}
$$

where $(-1)^{\tau}$ is the sign of a permutation $\tau$ of the symmetric group of degree 4.

In all that follows, unless stated otherwise, $R$ always denotes a prime ring, $Z(R)$ the center of $R, Q$ its Martindale quotient ring. The center of $Q$, denoted by $C$, is called the extended centroid of $R$ (we refer the reader to [1] for these objects). It is well-known that $C$ is a field. For any $x, y \in R$, the symbol $[x, y]$ and $x \circ y$ stand for Lie commutator $x y-y x$ and Jordan commutator $x y+y x$ respectively. An additive subgroup $U$ of $R$ is said to be a Lie ideal of $R$ if $[u, r] \in U$ for all $u \in U$ and $r \in R$. Recall that a ring $R$ is prime if for any $a, b \in R$, $a R b=(0)$ implies $a=0$ or $b=0$. An additive mapping $d: R \longrightarrow R$ is

(*) Indirizzo dell'A.: Department of Mathematics, Chuzhou University, Chuzhou Anhui, 239012, P. R. China.

E-mail:shulianghuang@sina.comｓhulianghuang@163.com 
called a derivation if $d(x y)=d(x) y+x d(y)$ holds for all $x, y \in R$. Starting from this definition, Brešar [2] introduced first the definition of generalized derivation: an additive mapping $F: R \longrightarrow R$ is called a generalized derivation if there exists a derivation $d: R \longrightarrow R$ such that $F(x y)=F(x) y+x d(y)$ holds for all $x, y \in R$, and $d$ is called the associated derivation of $F$. Hence, the concept of generalized derivations covers both the concepts of a derivation and of a left multiplier (i.e., an additive mapping satisfying $F(x y)=F(x) y$ for all $x, y \in R$ ). Basic examples are derivations and generalized inner derivations (i.e., mappings of type $x \longrightarrow a x+x b$ for some $a, b \in R$ ). We refer to call such mappings generalized inner derivations for the reason they present a generalization of the concept of inner derivations (i.e., mappings of the form $x \longrightarrow a x-x a$ for some $a \in R$ ). Zhang and Wang [20], defined first the concept of partial generalized automorphisms: an additive mapping $T: R \longrightarrow R$ is called a right (resp. left) partial generalized automorphism if there exists an automorphism $\sigma: R \longrightarrow R$ such that $T(x y)=$ $T(x) \sigma(y)$ (resp. $T(x y)=\sigma(x) T(y))$ holds for all $x, y \in R$. If $T$ is both left as well as a right partial generalized automorphism, then it is called a partial generalized automorphism. They proved that every partial generalized automorphism $T$ on a dense right ideal of $R$ can be uniquely extended to a partial generalized automorphism of $Q$ and assume the form $T(x)=\lambda \sigma(x)$ for all $x \in Q$ for some $\lambda \in C$ and an automorphism $\sigma$ of $Q$.

This paper is included in a line of investigation concerning the relationship between the global structure of a ring $R$ and the behaviors of some additive mappings defined on $R$ that satisfy certain special identities. A well-known result of Herstein [14] states that if $\rho$ is a right idea of $R$ such that $u^{n}=0$ for all $u \in \rho$, where $n$ is a fixed positive integers, then $\rho=0$. In [6], Chang and Lin considered the situation when $d(u) u^{n}=0$ for all $u \in \rho$, where $d$ is a nonzero derivation of $R$. In [8], Dhara and De Filippis studied the case when $u^{s} H(u) u^{t}=0$ for all $u \in L$, where $L$ a noncommutative Lie ideal of $R, H$ a generalized derivation of $R$ and $s, t$ are fixed nonnegative integers. More precisely, they proved the following: Let $R$ be a prime ring, $H$ a nonzero generalized derivation of $R$ and $L$ a noncommutative Lie ideal of $R$. Suppose that $u^{s} H(u) u^{t}=0$ for all $u \in L$. Then $R$ satisfies $s_{4}$, the standard identity in four variables.

On the other hand, in [5] Carini and De Filippis proved that if $R$ is a prime ring with $\operatorname{Char}(R) \neq 2$ satisfying $[d(u), u]^{n}=0$ for all $u \in L$, where $L$ is a noncentral Lie ideal and $d$ a nonzero derivation of $R$, then $R$ is commutative. 
De Filippis [7] generalized this result for generalized derivations. In [19], Wang studied the identity $[\sigma(u), u]^{n}=0$ replacing the derivation $d$ by an automorphism $\sigma$ of $R$ and obtained that $R$ satisfies $s_{4}$. Recently, Dhara and Sharma [9], considered the situation $\left(u^{n_{1}}[d(u), u] u^{n_{2}}\right)^{n_{3}}=0$ for all $u \in L$, where $n_{1}, n_{2}, n_{3}$ are fixed nonnegative integers, $L$ a noncentral Lie ideal and $d$ a derivation of $R$ and proved that $d=0$ provided $\operatorname{Char}(R) \neq 2$. Later, Dhara [10] extended this result by replacing the derivation $d$ with a generalized derivation $F$. Motivated by the previous results, we here prove a similar version for partial generalized automorphisms involving Jordan commutators. More precisely we will study the identity $\left(u^{s}(T(u) \circ u) u^{t}\right)^{n}=0$ for all $u \in L$, where $s \geq 0, t \geq 0, n \geq 1$ are fixed integers, $L$ a noncentral Lie ideal and $T \neq 0$ a partial generalized automorphism of $R$ and proved that $L$ is central in this case.

\section{Main results}

Theorem 2.1. Let $R$ be a prime ring, $T: R \longrightarrow R$ be a non-zero partial generalized automorphism of $R, I$ a two-sided ideal, $s \geq 0, t \geq 0$ and $n \geq 1$ fixed integers, such that $\left(u^{s}(T(u) \circ u) u^{t}\right)^{n}=0$ for all $u \in[I, I]$. If either $\operatorname{Char}(R)>n+1$ or $\operatorname{Char}(R)=0$, then $R$ is commutative.

Proof. Assume that $R$ is not commutative, so that $I$ is not central. In view of Zhang and Wang [20, Theorem 3.1], $T(x)=\lambda \sigma(x)$ for all $x \in R$, where $\lambda \in C$ and $\sigma$ is an automorphism of $R$. We are given that $\left(u^{s}(T(u) \circ u) u^{t}\right)^{n}=0$ for all $u \in[I, I]$. This implies that $\left([x, y]^{s}(\lambda \sigma([x, y]) \circ\right.$ $\left.[x, y])[x, y]^{t}\right)^{n}=0$ for all $x, y \in I$. Since $T$ is nonzero and so $\lambda \neq 0$, then it is invertible in $C$, which implies that $\left([x, y]^{s}(\sigma([x, y]) \circ[x, y])[x, y]^{t}\right)^{n}=0$ for all $x, y \in I$. If $\sigma=1_{R}$, the identity map on $R$, from above equation, $2^{n}[x, y]^{(s+t+2) n}=0$ and so $[x, y]^{(s+t+2) n}=0$ for all $x, y \in I$. Then it follows from Herstein [13, Theorem 2] that $R$ is commutative, a contradiction. Hence onward we assume that $\sigma \neq 1_{R}$. Since $\sigma$ is an automorphism of $R$, we have

$$
\left([x, y]^{s}([\sigma(x), \sigma(y)] \circ[x, y])[x, y]^{t}\right)^{n}=0 \text { for all } x, y \in I .
$$

By Kharchenko's theorem [16], we divide the proof into two cases.

CASE 1. Let $\sigma$ be $Q$-outer. Since either $\operatorname{Char}(R)>n+1$ or $\operatorname{Char}(R)=0$, by Chuang $\left[4\right.$, Main theorem], we arrive at $\left([x, y]^{s}([u, v] \circ[x, y])[x, y]^{t}\right)^{n}=0$ 
for all $x, y, u, v \in I$, in particular, by letting $u=x$ and $v=y$, then $2^{n}[x, y]^{(s+t+2) n}=0$ for all $x, y \in I$. By the same arguments as above, we get a contradiction.

CASE 2. If $\sigma$ is $Q$-inner, then there exists an invertible element $b \in Q$ such that $\sigma(x)=b^{-1} x b$ for all $x \in R$. We note that $b \notin C$ since $\sigma \neq 1_{R}$. By Chuang [3, Theorem 2], $I, R$ and $Q$ satisfy the same generalized polynomial identities (or GPIs in brief), from (2.1) we have

$$
\left([x, y]^{s}\left(\left[b^{-1} x b, b^{-1} y b\right] \circ[x, y]\right)[x, y]^{t}\right)^{n}=0 \text { for all } x, y \in I .
$$

In case the center $C$ of $Q$ is infinite, we have $\left([x, y]^{s}\left(\left[b^{-1} x b, b^{-1} y b\right] \circ\right.\right.$ $\left.[x, y])[x, y]^{t}\right)^{n}=0$ for all $x, y \in Q \otimes_{C} \bar{C}$, where $\bar{C}$ is the algebraic closure of $C$. Since both $Q$ and $Q \otimes_{C} \bar{C}$ are prime and centrally closed [12, Theorem 2.5 and Theorem 3.5], we may replace $R$ by $Q$ or $Q \otimes_{C} \bar{C}$ according as $C$ is finite or infinite. Thus we may assume that $R$ is centrally closed over $C$ (i.e., $R C=C$ ) which is either finite or algebraically closed and $\left([x, y]^{s}\left(\left[b^{-1} x b, b^{-1} y b\right] \circ[x, y]\right)[x, y]^{t}\right)^{n}=0$ for all $x, y \in R$. By Martindale [18, Theorem 3], $R C$ (and so $R$ ) is a strongly primitive ring. In light of Jacobson's theorem [15, pp.75], $R$ is isomorphic to a dense ring of linear transformations of a vector space $V$. Let ${ }_{R} V$ be a faithful irreducible left $R$ module with commuting division $D=\operatorname{End}\left({ }_{R} V\right)$. Since $C$ is either finite or algebraically closed, we know that $D$ must coincide with $C$. By the density theorem, $R$ acts densely on $V_{D}$.

For any given $v \in V$, we want to show that $v$ and $b v$ are linearly $D$ dependent. If $b v=0$ then $v$ and $b v$ are $D$-dependent and we are done in this case. Suppose that $b v \neq 0, v$ and $b v$ are $D$-independent. We consider the following two cases.

SubCase 1 . Assume that $v, b v, b^{-1} v$ are $D$-independent. Then by the density of $R$, there exist $x, y \in R$ such that

$$
x v=b v ; x b v=0 ; y v=0 ; y b v=v .
$$

From (2.2), we can see that

$$
0=\left([x, y]^{s}\left(\left[b^{-1} x b, b^{-1} y b\right] \circ[x, y]\right)[x, y]^{t}\right)^{n} v=(-1)^{(s+t+1) n} 2^{n} v \neq 0
$$

a contradiction.

SubCaSE 2. Otherwise, $v, b v, b^{-1} v$ are $D$-dependent. Since $v$ and $b v$ are $D$-independent, then $b^{-1} v=v d_{1}+b v d_{2}$ for some $d_{1}, d_{2} \in D$. Moreover, we claim that $d_{2} \neq 0$. Indeed, if $d_{2}=0$, then $b^{-1} v=v d_{1}$ and $v=b v d_{1}$, con- 
tradicting the independence of $v$ and $b v$. By the density of $R$, there exist $x, y \in R$ such that

$$
x v=0 ; x b v=v ; y v=b^{-1} v=v d_{1}+b v d_{2} ; y b v=b v d_{1} .
$$

It follows from (2.2) that

$$
0=\left([x, y]^{s}\left(\left[b^{-1} x b, b^{-1} y b\right] \circ[x, y]\right)[x, y]^{t}\right)^{n} v=2^{n} v d_{2}^{(s+t+2) n} \neq 0
$$

again a contradiction. From the above we have proven that $b v=v \alpha_{v}$ for all $v \in V$, where $\alpha_{v} \in D$ depends on $v \in V$. In fact, it is easy to check that $\alpha_{v}$ is independent of the choice of $v \in V$. Indeed, for any $v, w \in V$, by the above arguments, there exist $\alpha_{v}, \alpha_{w}, \alpha_{v+w} \in D$ such that $b v=v \alpha_{v} ; b w=w \alpha_{w}$; $b(v+w)=(v+w) \alpha_{v+w}$ and so $v \alpha_{v}+w \alpha_{w}=b(v+w)=(v+w) \alpha_{v+w}$. Hence $v\left(\alpha_{v}-\alpha_{v+w}\right)+w\left(\alpha_{w}-\alpha_{v+w}\right)=0$. If $v$ and $w$ are $D$-independent, then $\alpha_{v}=\alpha_{v+w}=\alpha_{w}$ and we are done. Otherwise, $v$ and $w$ are $D$-dependent, say $v=\lambda w$ for some $\lambda \in D$. Thus $v \alpha_{v}=b v=b \lambda w=\lambda b w=\lambda w \alpha_{w}=v \alpha_{w}$, that is $V\left(\alpha_{v}-\alpha_{w}\right)=0$. Since $V$ is faithful, hence $\alpha_{v}=\alpha_{w}$.

So we conclude that there exists $\delta \in D$ such that $b v=v \delta$ for all $v \in V$. We claim that $\delta \in Z(D)$, the center of $D$. Indeed, for any $\beta \in D$, we have $b(v \beta)=(v \beta) \delta=v(\beta \delta)$ and on the other hand $b(v \beta)=(b v) \beta=(v \delta) \beta=v(\delta \beta)$. Therefore $V(\beta \delta-\delta \beta)=0$ and hence $\beta \delta=\delta \beta$, which implies that $\delta \in Z(D)$. So $b \in C$, a contradiction. This completes the proof.

REMARK. Let $R$ be a prime ring and $L$ a non-central Lie ideal of $R$. Then either there exists a non-zero ideal $I$ of $R$ such that $0 \neq[I, R] \subseteq L$ or $\operatorname{Char}(R)=2$ and $R$ satisfies $s_{4}$.

Proof. See [14, pp. 4-5], [11, Lemma 2] and [17, Theorem 4].

TheoRem 2.2. Let $R$ be a prime ring with center $Z(R), T: R \longrightarrow R$ be a non-zero partial generalized automorphism of $R, L$ a Lie ideal of $R$, $s \geq 0, t \geq 0$ and $n \geq 1$ fixed integers, such that $\left(u^{s}(T(u) \circ u) u^{t}\right)^{n}=0$ for all $u \in L$. If either $\operatorname{Char}(R)>n+1$ or $\operatorname{Char}(R)=0$, then $L \subseteq Z(R)$.

Proof. Assume that $L$ is non-central. By previous Remark and since $\operatorname{Char}(R) \neq 2$, there exists a non-zero ideal $I$ of $R$ such that $0 \neq[I, R] \subseteq L$. By Theorem 2.1, it follows that $R$ is commutative, which is a contradiction.

The following example demonstrates that $R$ to be prime is essential in Theorem 2.1. 
ExAmple 2.3. Let $Z$ be the ring of all integers. Set $R=\left\{\left(\begin{array}{ccc}0 & a & b \\ 0 & 0 & c \\ 0 & 0 & 0\end{array}\right) \mid a, b, c \in Z\right\}$ and $I=\left\{\left(\begin{array}{ccc}0 & a & b \\ 0 & 0 & 0 \\ 0 & 0 & 0\end{array}\right) \mid a, b \in Z\right\}$. Next, let us define a mapping $T: R \longrightarrow R$ given by $T\left(\begin{array}{lll}0 & a & b \\ 0 & 0 & c \\ 0 & 0 & 0\end{array}\right)=$ $\left(\begin{array}{ccc}0 & -a & b \\ 0 & 0 & -c \\ 0 & 0 & 0\end{array}\right)$. The fact $\left(\begin{array}{ccc}0 & 1 & 0 \\ 0 & 0 & 0 \\ 0 & 0 & 0\end{array}\right) \neq 0$ implies that $\left(\begin{array}{lll}0 & 1 & 0 \\ 0 & 0 & 0 \\ 0 & 0 & 0\end{array}\right)$ $\left(\begin{array}{lll}0 & a & b \\ 0 & 0 & c \\ 0 & 0 & 0\end{array}\right)\left(\begin{array}{lll}0 & 1 & 0 \\ 0 & 0 & 0 \\ 0 & 0 & 0\end{array}\right)=0$, proving $R$ is not prime. And it is clear that

$I$ is a non-zero ideal of $R$ and $T$ is a nonzero partial generalized automorphism of $R$. And it is easy to check that $\left(u^{s}(T(u) \circ u) u^{t}\right)^{n}=0$ for all $u \in[I, I]$. However $R$ is not commutative.

Acknowledgments. The author is greatly indebted to the referee for pointing out an error in the original manuscript and some useful suggestions on how to arrange the paper.

This research was supported by the Natural Science Research Foundation of Anhui Provincial Education Department (No. KJ2012B125) and also by the Anhui Province College Excellent Young Talents Fund Project (No. 2012SQRL155) of China.

\section{REFERENCES}

[1] K. I. Beidar - W. S. Martindale - V. Mikhalev, Rings with generalized identities, Monographs and Textbooks in Pure and Applied Mathematics, 196. Marcel Dekker, Inc., New York, 1996.

[2] M. BREŠAR, On the distance of the composition of two derivations to be the generalized derivations, Glasgow Math. J., 33 (1991), pp. 89-93.

[3] C. L. Chuang, GPIs having coefficents in Utumi quotient rings, Proc. Amer. Math. Soc., 103 (3) (1988), pp. 723-728.

[4] C. L. Chuang, Differential identities with automorphisms and anti automorphisms, J. Algebra, 160 (1993), pp. 130-171.

[5] L. CARINI - V. DE FILIPPIS, Commutators with power central values on a Lie ideal, Pacific J. Math., 193 (2) (2000), pp. 269-278.

[6] C. M. Chang - Y. C. Lin, Derivations on one-sided ideals of prime rings, Tamsui Oxf. J. Math. Sci., 17 (2) (2001), pp. 139-145.

[7] V. DE FILIPPIS, Generalized derivations and commutators with nilpotent values on Lie ideals, Tamsui Oxf. J. Math. Sci., 22 (2006), pp. 167-175. 
[8] B. Dhara - V. De FilipPis, Notes on generalized derivations on Lie ideals in prime rings, Bull. Korean Math. Soc., 46 (3) (2009), pp. 599-605.

[9] B. Dhara - R. K. Sharma, Derivations with power central values on Lie ideals in prime rings, Czech. Math. J., 58 (2008), pp. 147-153.

[10] B. DHARA, Generalized derivations with vanishing power values on Lie ideals (to appear).

[11] O. M. Di Vincenzo, On the n-th centralizers of a Lie ideal, Boll. U. M. I., 3-A (1989), pp. 77-85.

[12] J. S. Erickson - W. S. Martindale III - J. M. Osborn, Prime nonassociative algebras, Pacific J. Math., 60 (1) (1975), pp. 49-63.

[13] I. N. Herstein, Center-like elements in prime rings, J. Algebra, 60 (1979), pp. $567-574$.

[14] I. N. Herstein, Topics in ring theory, Univ. of Chicago Press, Chicago, 1969.

[15] N. Jacobson, Structure of rings, Amer. Math. Soc., Providence, RI, 1964.

[16] V. K. Kharchenko, Generalized identities wtih automorphisms, Algebra i Logika, 14 (1975), pp. 132-148.

[17] C. Lanski - S. Montgomery, Lie structure of prime ring of characteristic 2, Pacific J. Math., 42 (1) (1972), pp. 117-136.

[18] W. S. Martindale III, Prime rings satisfying a generalized polynomial identity, J. Algebra, 12 (1969), pp. 176-584.

[19] Y. WANG, Power-centralizing automorphisms of Lie ideals in prime rings, Comm. Algebra, 34 (2006), pp. 609-615.

[20] X. ZHANG - Y. WANG, (Right) partial generalized automorphisms of semiprime rings, Northeast Math. J., 18 (3) (2002), pp. 261-265.

Manoscritto pervenuto in redazione il 20 Settembre 2011. 
